\title{
AN HMV MAPPING STUDY OF A PULSATING JET SYSTEM
}

Jekaterina Kuleshova ${ }^{\mathrm{a}}$, Peter R. Birkin ${ }^{*}$ and Joanne M. Elliott ${ }^{\mathrm{b}}$

a School of Chemistry, University of Southampton, Southampton, SO17 1BJ United Kingdom

${ }^{b}$ School of Chemistry, University of Reading, Reading, United Kingdom

\section{Abstract}

A new system for the generation of hydrodynamic modulated voltammetry (HMV) is presented. This system consists of an oscillating jet produced through the mechanical vibration of a large diaphragm. The structure of the cell is such that a relatively small vibration is transferred to a large fluid flow at the jet outlet. Positioning of an electrode ( $\mathrm{Pt}, 0.5 \mathrm{~mm}$ or $25 \mu \mathrm{m}$ diameter) over the exit of this jet enables the detection of the modulated flow of liquid. While this flow creates modest mass transfer rates (time averaged $\sim 0.015 \mathrm{~cm} \mathrm{~s}^{-1}$ ) it can also be used to create a HMV system where a 'lock - in' approach is adopted to investigate the redox chemistry in question. This is demonstrated for the $\mathrm{Fe}(\mathrm{CN})_{6}{ }^{3-14-}$ redox system. Here 'lock - in' to the modulated hydrodynamic signal is achieved through the deployment of bespoke software. The apparatus and procedure is shown to produce a simple and efficient way to obtain the desired signal. In addition the spatial variation of the HMV signal, phase correction and time averaged current with respect to the jet orifice is presented.

\section{Keywords: hydrodynamic modulation; jet; electrochemistry; microelectrode; mapping}

\footnotetext{
* Corresponding author, prb2@soton.ac.uk, tel: +44 2380 594172, fax: +44 2380593781
} 


\section{Introduction}

The detection of trace species within a solution is of clear analytical importance. A number of electroanalytical systems have been developed with the aim of investigating sub micromolar levels of important analytes [1]. Examples of such techniques are stripping voltammetry, pulse voltammetric techniques $[2,3]$ and hydrodynamic modulated systems [4]. In the last of these techniques a particular experimental protocol is developed which results in a periodically altering mass transfer regime. The method usually relies on a lock-in approach to target the mass transport dependant signal from electrochemically active species within the solution phase. Other processes, such as charging of the double layer and surface activity, which do not posses a mass transfer component, are hence eliminated from the system. In this way the voltammetric response of a system is refined to focus only on the mass transfer dependant components. Clearly this is a useful technique for distinguishing trace analytes on electrodes with high non mass transfer dependent electrochemical processes (e.g. modified electrodes or electrodes with high surface areas). Early examples include a modified rotating disk experiment whereby the frequency of rotation of the disk is modulated at an appropriate level [5-11]. Employing this technique, detection limits of the order of $0.05 \mu \mathrm{M}$ have been reported. Other examples of these systems involve, pulsed rotation [12-15], vibrating electrode techniques $[16,17]$, ultrasonic cavitation $[18,19]$ or, more recently, vibrating or chopped capillary flow systems based around a novel scanning electrochemical microscopy (SECM) technique [20-22]. In all of these cases the detection limits proposed have been impressive, generally below 0.5 $\mu \mathrm{mol} \mathrm{dm}{ }^{-3}$. An excellent review of the pertinent literature can also be found [4]. While all of these systems have merit, they have generally been limited to the 
modulation of the rotation rate of an RDE electrode, the oscillation [16] of a continuously flowing jet or the vibration of an electrode. Here we present a new method for generating an oscillatory flow and then its subsequent deployment to investigate HMV information as a function of experimental variables such as electrode potential and electrode position. In this case an oscillating hydrodynamic jet is developed and tested. The system presented consists of a membrane of relatively large cross sectional area which is driven into oscillation by an appropriate mechanical shaker. The displaced volume is then concentrated through an appropriately narrowing glass section. This results in a relatively small membrane oscillation being transposed to a high amplitude liquid pulsation at the exit of the jet. This pulsating motion is controlled by the frequency and amplitude of the membrane vibration. An electrode is then mounted above the system and in the presence of a suitable redox active species, can be used to monitor the periodic flow induced by the apparatus Note that the study of oscillating jets has been detailed in the literature and examples of the flow and vortex behaviour of various exit geometries using tracer methods [23] can be found in the literature [24, 25]. However, in the system deployed here, the oscillation of flow is bipolar in nature as the liquid is periodically ejected and then drawn back into the body of an apparatus. In addition we use this oscillating system to investigate its flow characteristics and its possible deployment for HMV studies. Lastly, as subsequently be demonstrated, the system has the ability to be used in a HMV mode, to measure the phase angle between the reference and current time signals or as a direct 'time averaged' hydrodynamic system. The results of this study will now be detailed. 


\section{Experimental}

The experimental arrangement used in this work is shown in Fig. 1. This consists of a $\sim 3.5 \mathrm{~cm}$ radius membrane attached to the base of an inverted funnel. The funnel neck is then pulled into a $2 \mathrm{~mm}$ diameter jet orifice. A mechanical shaker (LDS type V101 mini shaker) was attached to the centre of the membrane with a mechanical coupling incorporating a single axis accelerometer (Model 3100B, Dytran Instruments, Inc., sensitivity $99.3 \mathrm{mV} \mathrm{g}^{-1}$ ). The accelerometer signal was conditioned with an amplifier (Model 4105C, Dytran Instruments, Inc., with voltage gains of $x 1, x 10, x 100$ ) and recorded on an oscilloscope (Techtronixs, TDS 2014, 1GS/s, $100 \mathrm{MHz}$ ). The shaker was driven by an amplifier (Model AX-220, JVC) which is in turn supplied by a function generator (Model FG 100, Digimess). In all cases the frequency of oscillation of the membrane, and hence jet, was $16 \mathrm{~Hz}$. The electrodes employed (Pt, $0.5 \mathrm{~mm}$ and $25 \mu \mathrm{m}$ diameters) were fabricated in-house and were sealed in glass (diameter $5.0 \mathrm{~mm}$ for the $0.5 \mathrm{~mm}$ electrode and $2 \mathrm{~mm}$ or $5.5 \mathrm{~mm}$ for the $25 \mu \mathrm{m}$ electrode). Positioning of the electrode with respect to the jet orifice was controlled using an XYZ stage (Zaber, $60 \mathrm{~mm}$ travel, resolution 0.1 $\mu \mathrm{m}$, TLA 60A actuators connected to TSB 60-M stages). The electrodes were hand polished to a mirror like finish, in a conventional manner, using alumina powder slurries (Struers, alumina $0.3 \mu \mathrm{m}$ diameter) on microcloth (Buehler) polishing pads. A two electrode system (using a silver wire as a pseudo reference/counter) was employed in the experiments consisting of in-house constructed equipment interfaced to a PC through an ADC card (Computer boards type PCI-DAS1602/16). Software, to determine either conventional cyclic voltammetry or the HMV signal, was developed in-house. A Photron APX RS high-speed camera with a Navitron lens (12x Zoom, with lens attachment 
$0.5 \mathrm{x}$ and $0.5 \mathrm{x}$ adapter) was used to capture the images of fluid motion within the cell. In order to acquire the images, the apparatus was back lit by a High bright white LED source (PHLOX-LEDW-BL-50x50-S-Q-1R-24V). Image analysis was achieved through the use of Photron Motion Tools Software.

Solutions were prepared using a Purite Select Fusion 160 (Ondeo) water purification system (resistivity typically $18.2 \mathrm{M} \Omega \mathrm{cm}$ and a TOC $<10 \mathrm{ppb}$ ).

$\mathrm{Sr}\left(\mathrm{NO}_{3}\right)_{2}$ (Aldrich, 99+\%), $\mathrm{K}_{3}\left[\mathrm{Fe}(\mathrm{CN})_{6}\right]$ (Sigma, 99.5\%), Heptane (Alfa Aesar, 99\%) and red food colouring (Super Cook) were employed as received. 


\section{Results and Discussion}

The experimental system deployed (shown in figure 1) was investigated using both high-speed camera analysis and electrochemical investigations. Figure 1 shows that the minishaker is connected to the membrane via a mechanical coupling incorporating an accelerometer. The inclusion of the accelerometer into the design was to enable calibration and monitoring of the motion of the membrane during the experiments. In order to calculate the displacement of the centre of the membrane (coupled to the mechanical shaker), the motion is assumed (see later data) to be largely sinusoidal in nature. Hence it is possible to calculate the zero to peak amplitude of the membrane centre from the accelerometer output. In order to do this, the zero to peak acceleration amplitude derived from the accelerometer output $\left(A, \mathrm{~m} \mathrm{~s}^{-2}\right)$ can be converted to the displacement amplitude of the membrane centre $(X, \mathrm{~m})$ by the employment of equation (1)

$$
X=A /(2 \pi f)^{2}
$$

where $f$ represents the frequency $(\mathrm{Hz})$ of oscillation of the shaker employed in the experiment. In order to validate the motion of the components (for example the fluid motion and the action of the membrane and shaker) within the experimental apparatus employed, high-speed camera analyses were performed. Figure 2(a) shows a sequence of frames recorded at $500 \mathrm{fps}$ as the membrane of the cell was vibrated at $16 \mathrm{~Hz}$. In this case the motion of the fluid within the neck of the funnel is shown. In order to do this the lower section of the funnel was filled with water containing a red dye while the upper section was filled with heptane. This produced a liquid/liquid interface in the neck of the funnel which could be easily followed using the high-speed camera employed. Note that attempts to monitor the fluid motion in the absence of the heptane 
layer proved unsuccessful as the liquid/air interface broke up during oscillatory motion at the higher amplitudes employed. If the motion of the membrane is assumed to be ideal (so that at maximum extension a conical section is developed), then the fluid velocity in the neck or at the jet orifice itself, can be calculated. Using this assumption the volume displaced $(V)$ by the shaker motion (in this case over a complete oscillation of the membrane) is given by equation (2), $V=2 / 3 \pi r_{m}{ }^{2} X$

where $r_{m}$ represented the membrane radius (in this case $35 \mathrm{~mm}$ ). This volume displacement can be related to the velocity amplitude at the jet exit through the equivalent volume at this point. Hence the jet zero to peak amplitude velocity $\left(v_{j e t}\right)$ is given by equation (3),

$v_{j e t}=\omega\left[\frac{1 / 3 r_{m}^{2} X}{r_{j}^{2}}\right]$

where $r_{j}$ represents the jet radius (in this case $1 \mathrm{~mm}$ ) and $\omega$ the angular frequency ( $\omega=2 \pi f$, where $f$ represents the frequency in $\mathrm{Hz}$ of the membrane vibration) of membrane vibration. Considering the membrane displacement amplitudes typically employed (measured by the accelerometer at $\sim 0.3 \mathrm{~mm}$ ) and the frequency and dimensions of the system employed, the jet amplitude velocity is of the order of $12.3 \mathrm{~m} \mathrm{~s}^{-1}$. Clearly this calculation assumes ideal behaviour from the membrane. In order to test this assumption, the motion of the liquid within the neck of the apparatus (see figure 1) was recorded using a high-speed camera. Figure 2(b) shows the actual amplitude of oscillation of a liquid/liquid interface within the neck assuming that the shape of the meniscus can be approximated as an oblate spheroid [26]. In addition the predicted displacement amplitudes are shown using the accelerometer to predict the 
motion of the membrane. Under the conditions employed, displacement amplitudes of $\sim 30 \mathrm{~mm}$ are expected in the neck of the inverted funnel. However, the high-speed camera experiments clearly show that the actual displacement is of the order of $2.5 \mathrm{~mm}$. This is clearly significantly less than expected. This suggests that the assumption of ideal behaviour of the membrane is not maintained under the conditions employed. This cannot be accounted for by errors associated with the accelerometer measurements. Indeed the motion predicted by the accelerometer was validated by measuring the displacement amplitude of this component with a high-speed camera and then comparing this value $(0.82 \mathrm{~mm})$ to the displacement amplitude predicted by the accelerometer output $(0.88 \mathrm{~mm})$. Clearly this cannot account for the observed deviation in figure 2(b). However, imaging the membrane itself provides strong evidence that the deviation between the predicted and measured displacements shown in figure 2(b) was as the result of non-ideal motion of the membrane. These images (not shown) revealed that the membrane does not operate in an ideal fashion. Indeed as the centre of the membrane is displaced by the mechanical transducer, the outer section of the circular membrane moves down (presumably due to the forces resulting from the fluid inertia and backpressure associated with flow [27] through the jet). This significantly reduces the displaced volume, which in this case appears to be an order of magnitude lower than expected. Nevertheless, significant periodic flow rates are generated by this simple apparatus and, as will be subsequently demonstrated, this motion allows for HMV analysis to be undertaken.

In order to analyse the motion of the fluid at the exit of the jet, a series of experiments were performed using an electrode to measure the mass transfer 
limited current for the reduction of $\mathrm{Fe}(\mathrm{CN})_{6}{ }^{3-}$. In order to maintain mass transfer limited conditions the potential of the electrode was held at $-0.2 \mathrm{~V}$ vs. Ag during this experiment. Figure 3 shows the current time history for a $500 \mu \mathrm{m}$ diameter Pt electrode positioned above the jet outlet under conditions of oscillation. Included on the figure is the signal from the function generator which is used as a reference signal for lock in measurements (see later discussion) and the output from the accelerometer. First, note that the pulsating motion of the liquid at the funnel mouth is detected as a transient enhancement (in this case up to $2.5 \times 10^{-2} \mathrm{~cm} \mathrm{~s}^{-1}$ ) in current which is largely asymmetric in nature but occurs at the same frequency as the pulsation driven by the shaker (here $16 \mathrm{~Hz}$ ). Clearly, as the fluid flows out and into the jet mouth, one would expect current enhancement as a result of fluid flow in the two directions. Indeed the current time transients shown in figure 3 have a large cathodic transient (labelled ' $A$ ') followed by a smaller ridge in the decay of this transient (see label 'B') Hence, for this relatively large electrode it appears that the current is dominated by one of the flow directions. As a result of this, the pulsation frequency of the current recorded at the electrode is the same as the membrane driver. Further experiments are currently underway to determine the major flow contribution to the current pulses. Interestingly, when an identical experiment was performed with a $25 \mu \mathrm{m}$ diameter Pt microelectrode, evidence for both flows providing enhanced current time transients is gathered. However, one of the flows still plays a more significant role. Second, there is a significant phase difference between the reference signal (here a $1 \mathrm{~V}$ amplitude supplied to the amplifier prior to the shaker) and the peak of the current time data. Lastly, figure 3 shows that the accelerometer data, although largely sinusoidal in nature, contains non-idealised motion. It should be noted that this non-ideality was 
found to be highly dependent on the mechanical integrity of the coupling between the shaker and the membrane and framework used to construct the cell.

In order to characterise the electrochemical HMV signal obtained from the pulsating jet apparatus described above, bespoke software was developed to capture the data and extract the amplitude of the HMV signal as a function of the electrode potential, time or 3D position of the electrode with respect to the jet mouth. This software relied on an ADC card (sampling in this case at $10 \mathrm{kHz}$ over a $0.5 \mathrm{~s}$ time window) and then signal processing of the data (correcting for phase shifts etc.) to extract the relevant data [28]. This approach is similar to the deployment of a conventional lock in amplifier but with the advantage of introducing dynamic phase correction, DC background current subtraction and integration of the HMV analysis with the electrochemical experiments employed. Figure 4 shows a schematic representation of the procedure adopted to capture and analyse the data. This 'virtual lock in' procedure (the system is run in VB on a PC with an ADC card) relies on two basic procedures. In the first procedure the electrode is held at the mass transfer limited potential for the redox species in question and a set of data is captured over a $0.5 \mathrm{~s}$ period. In order to avoid DC offsets, the data set is averaged to attain the time averaged signal ( $\left.\mathrm{i}_{\text {av }}\right)$ which is then subtracted from the raw data to leave the AC signal. At this stage the phase angle is determined by varying the temporal position of the reference signal (gathered at the same time as the experimental data) until a maximum or minimum in the data analysis is determined. This phase shift, which was found to be constant as long as the electrode was maintained in one position in the cell, is then used to determine the HMV signal as a function of electrode potential. In the second procedure (where the electrode position is 
varied while the electrode potential was held at the mass transfer limited potential for the redox system employed) a different analysis routine was deployed. Here, the data is gathered and the time averaged current calculated and subtracted from the raw experimental data. The phase angle to give the maximum HMV signal were then determined. Hence three experimental parameters were determined as a function of the electrode position. These were the time averaged current, the phase angle to give the maximum HMV amplitude (in the case of the $\left[\mathrm{Fe}(\mathrm{CN})_{6}\right]^{3-}$ redox species, most negative) and the HMV amplitude itself. The electrode could then be moved to a different position and the process repeated. An example of how the HMV signal can be determined as a function of electrode potential is shown in figure 5 . This shows the cyclic HMV signal recorded for a $5 \mathrm{mmol} \mathrm{dm}^{-3}$ solution of $\left[\mathrm{Fe}(\mathrm{CN})_{6}\right]^{3-}$ solution recorded at a $500 \mu \mathrm{m}$ diameter Pt electrode. Clearly the HMV signal correlates closely ${ }^{\dagger}$ with the amplitude of the current oscillation (see figure 3 ) as expected. However, at this concentration level the DC (time averaged) signal directly from the electrode shows a clear response to the flow of liquid as a result of the pulsating jet. Figure 5 also shows that the response of the electrode is markedly different in the absence of jet action. The time averaged signal ( $\left.\mathrm{i}_{\mathrm{av}}\right)$ shows a clear classic cyclic voltammetric response of a macroelectrode while the lock in signal (i $\left.\mathrm{i}_{\mathrm{HMV}}\right)$ shows no activity over the entire voltage range investigated. This demonstrates the ability of the lock in approach to discriminate between the desired signal and other components not at the reference frequency. Clearly this demonstrates the potential advantage

\footnotetext{
† Note the value of the HMV signal will not match exactly the amplitude of the current transient induced by the jet as this analysis is based on matching sinusoidal signals. Here the transient is clearly not sinusoidal. However, the amplitude is 80-90 \% (see figure 5 for cyclic HMV data) of that expected considering a pure sinusoidal response of the electrode as $100 \%$.
} 
of using this type of analysis under the conditions employed and highlights the use of HMV in the study of mass transfer dependant reactions at conventional electrodes. Further investigation of the potential of this particular flow modulation for detection of trace species is currently underway.

As well as the conventional detection of mass transfer dependant redox active species within a solution, it is possible to use the HMV approach, described here, to study the fluid motion through the jet mouth itself (as a function of the position of the electrode with respect to the jet orifice). Here a number of different experiments were performed with a range of electrode sizes (with variable active geometric electrode areas and support dimensions). Two active electrode areas were deployed: a set of $25 \mu \mathrm{m}$ diameter Pt electrodes and a $0.5 \mathrm{~mm}$ diameter Pt electrode. For these experiments two programs (the software driven lock-in and a xyz - stage controller) were combined. This combination enabled the position of the electrode to be controlled while simultaneously recording the experimental HMV signal. However, it should be noted that as the electrode moves with respect to the jet, the phase angle between the reference signal and the electrochemical signal was observed to change. Finally it is also possible to calculate the time averaged DC signal ( $\left.\mathrm{i}_{\mathrm{av}}\right)$ at each electrode position. Figure 6 shows a set of results for a $25 \mu \mathrm{m}$ diameter Pt electrode as the electrode was scanned over the region above the jet in a raster like fashion. Note that the HMV signal ( $\left.\mathrm{i}_{\text {HMV }}\right)$ and the phase angle used to obtain this value are recorded as a function of position of the electrode employed in the xy plane above the jet orifice (at a constant $z$ displacement of 1 $\mathrm{mm}$ ). Figure 6(a) shows that moving the electrode away from the centre of the jet altered the phase angle between the reference and experimental signals. Indeed the phase angle is closely associated to the size of the jet itself and 
shows a phase angle close to zero within this zone. The diameter of this zone is $\sim 3 \mathrm{~mm}$. This matches the sum of the diameter of the jet $(2 \mathrm{~mm})$ and the radius of the electrode support $(1 \mathrm{~mm})$. It seems that when the electrode centre moves out of this zone, a major shift in the phase angle is detected. The electrode surround clearly influences the detected phase (and as will be shown subsequently, the spatial resolution of the HMV signal). This suggests that the liquid from the jet is 'deflected' across the surface of the electrode support. Hence, even if the electrode (here a $25 \mu \mathrm{m}$ diameter Pt disk) is outside of the jet, deflection of fluid along the surface of the glass body results in a detectable signal. Figure 6(b) supports this conclusion as it shows that the amplitude of the HMV signal is greatest around the mouth of the jet (in this case $2 \mathrm{~mm}$ in diameter) but the signal can be seen to extend to greater distances before returning to the background level. Clearly figure 6 (a) and (b) show that outside of the jet, both the phase angle and the HMV signal change dramatically. Far from the jet the HMV signal falls to -2 nA from a peak value of approximately $23 \mathrm{nA}$. It is also apparent that the microelectrode shows an increased response around the edge of the mouth of the jet. In these positions a ring of maximum HMV signal, which surrounds a central circular area where the experimental signal is slightly lower, was observed. A similar effect has been previously reported by Macpherson et al. when investigating the signal from a wall tube electrode arrangement [29, 30]. They suggested that as an electrode is moved from the centre of the jet, convective flow in the stagnation region has contributions from both axial and radial velocity components. However, it should be noted that the experimental system employed here and that of Macpherson et al., although both hydrodynamic in nature, are very different. Here we employ a pulsating flow where the periodicity and flow direction is 
governed by the vibration of a diaphragm. However, Macpherson et al. employed a modulated flow from a fine capillary positioned over a microelectrode. Hence, direct comparisons are not appropriate. However, the increased HMV signal observed around the mouth of the jet wall, in the experiments reported here, would also suggest that this region is of significance for this pulsating system. One further note should be highlighted; in this pulsating system (and the associated HMV approach) the phase angle detected does not seem to detect this 'dimple' zone in the centre of the jet. This indicates that it is the amplitude of the signal that varies in this region alone rather than a combination of effects. Lastly the time averaged signal is presented in figure 6 (c). Again a clear enhancement in the current detected at the microelectrode is seen. However, the definition around the jet using this averaged signal is less than that observed using the HMV lock in mode. Hence the current does not drop directly to the background level when the electrode moves out of the flow driven directly by the pulsating jet. This is understandable as general forced convection will contribute to the time averaged ( $\left.\mathrm{i}_{\mathrm{av}}\right)$ signal detected by the microelectrode.

In order to show the influence of the electrode support on the HMV signal detected at a microelectrode, an electrode with a larger support was employed. In this case a $25 \mu \mathrm{m}$ diameter Pt microelectrode with insulating sheath radius of $5.5 \mathrm{~mm}$ (significantly larger than the size of funnel mouth) was used. Figure 7 (a) shows the phase angle measurement determined in this experiment where the electrode was scanned over the mouth of the pulsating jet at a constant height of $1 \mathrm{~mm}$ in the xy plane. Again the centre of the jet appears as a phase angle (to generate the highest amplitude of the HMV signal) close to zero degrees. However, at greater distances a 'halo' effect was observed. Again it 
appears that the radius of the electrode support (here $2.75 \mathrm{~mm}$ ) in combination with the jet radius $(1 \mathrm{~mm}$ ) contributes to the ring of phase activity (radius 3.75 $\mathrm{mm}$ ) seen in the image. It would appear that this effect is the result of the flow profiles generated by the pulsating flow and its interaction with the body of the electrode. However, further discussion of this effect is beyond the scope of this paper and more investigations into this effect are currently underway. Figure 7 (b) shows the HMV amplitude as a function of the xy position of the electrode with respect to the jet orifice (here located at $y \sim 2 \mathrm{~mm}, \mathrm{x} \sim 0 \mathrm{~mm}$ ). Again a ring like HMV signal was observed around the funnel mouth. However, figure 7 also shows that with this bigger electrode surround, a second ring of increased current appears outside the funnel mouth. The radius of this 'ridge' is $\sim 3.75$ $\mathrm{mm}$ closely matching the sum of the radius of the electrode support and jet. This suggests that even after crossing the boundary of the funnel mouth, flow from the jet is deflected to the microelectrode surface, where it is detected as an increased electrochemical signal. Interestingly this deflection is seen as a current ridge for this size of electrode support whereas the smaller electrodes (see figure 6) does not observe this phenomena. Lastly the time averaged current response is plotted as function of xy position in figure 7 (c). This again demonstrates that the resolution of this approach is less than that found when employing the HMV analysis (which shows enhances definition around the jet mouth, see figure 7 (b)). Figure 7 (b) and 7 (c) show the clear 'dimple' effect at the centre of the jet exit. However, the enhanced activity around the jet mouth is only shown clearly with the HMV lock in data analysis approach (see figure 7 (b)).

While the experimental results shown in figure 6 and 7 clearly show the spatial extent of the signals detected in the xy plane, they give no indication of 
the extent of this enhanced jet edge effect as a function of the vertical distance away from the jet mouth. Hence further experiments were performed to investigate this behaviour. Figure 8 shows the vertical extent of the jet edge effect and the influence of the size of the electrode support. Here the current profile is mapped while the microelectrodes were moved in the vertical (xz) plane (note the y position was manually adjusted prior to the scan to ensure that the electrode mapped the region of interest bisecting the diameter of the jet mouth). Figure 8 clearly indicates that the maximum current enhancement is smallest when $z$ (vertical) displacement is greatest as expected if the flow from the jet dissipates into the solution. However, this decrease in current for the smaller diameter electrode support (see figure $8(a)$ ) is more dramatic when compared to the larger electrode support (see figure 8 (b)). This supports the assertion that the electrode surround has a significant effect on the experimental results. The larger the electrode surround, the greater the interaction with the fluid flow from the jet (and subsequent reduction in spatial resolution of the electrode) towards the $25 \mu \mathrm{m}$ diameter microelectrode is observed. In addition figure 8 shows jet edge effects as two zones of high activity extending away (up to $\sim 1 \mathrm{~cm}$ ) from the edge of the jet. This supports the results presented in figure 6 and 7 which indicate that the HMV signal was greatest at the edge of the jet mouth.

Similar scanning experiments have been performed using a $500 \mu \mathrm{m}$ diameter Pt electrode sealed in a $5.0 \mathrm{~mm}$ diameter glass support. Figure 9 (a), (b) and (c) show the phase angle, HMV and time averaged signal respectively as a function of the xy position of the electrode (note in this case the centre of the jet of $x \sim 0 \mathrm{~mm}$ and $\mathrm{y} \sim 0 \mathrm{~mm}$ ). Figure 9 (a) shows the phase correction (to maintain the highest HMV amplitude) plotted as a function of the xy position as 
the electrode was scanned in the xy plane at constant $z$ displacement $(1 \mathrm{~mm})$. This shows a distinct relationship between phase angle and the location of the electrode. At the centre of the jet the phase correction is $\sim 200^{\circ}$. This is significantly greater that that observed for the microelectrode (see figure 6 and 7). However, this is to be expected as the response time of this $500 \mu \mathrm{m}$ diameter electrode is larger than the $25 \mu \mathrm{m}$ diameter microelectrode. Figure 9 (b) clearly shows that the $500 \mu$ m diameter electrode produces a well defined HMV signal from the pulsating jet with the added consequence of the larger electrode producing an enhanced current signal. As expected, due to both temporal and spatial resolution issues, the current enhancement around the lip of the jet exit is very regular and does not show the variation detected by the 25 $\mu \mathrm{m}$ diameter Pt electrode employed previously (see figure 6 and 7).

Presumably the smaller electrode can detect greater variation in the flow pattern emanating from the jet orifice. Lastly the average current, although still showing evidence of the 'dimple' in the centre of the jet, has a significantly poorer spatial resolution with the enhanced current zone extending completely across the area scanned (see figure 9 (c)). This is to be expected as the time averaged signal has none of the temporal information captured in the HMV data. In turn any flow from the jet is detected as an enhanced current signal due to forced convection in the cell. The HMV signal shown in figure $9(\mathrm{a})$ is clearly well defined and very symmetrical in nature. Indeed, more so than the corresponding microelectrode experiments (see figure 6 and 7 ). However, this may be a consequence of the size of the electrode smoothing out any flow variations produced by the jet action itself.

Finally the results presented in figures 6,7 and 9 indicate that, depending on the choice of measurement criteria (HMV, average current or 
phase angle employed) selected, the spatial resolution and information gathered by the experiments can vary dramatically. The HMV analysis clearly shows the 'ring' effect from the jet edge most clearly while the averaged current gives the poorest resolution. Interestingly the phase angle correction data shows no evidence for the ring effect from the jet edge although still showing evidence for the influence of the electrode support.

\section{Conclusions}

An HMV system using a mechanically oscillated pulsating jet has been developed. High-speed imaging of the fluid flow within the system shows the periodic and amplified fluid motion with the apparatus. The pulsation of the jet has been successfully employed with a software based 'lock-in' approach to capture HMV signals of a simple redox system. Improved spatial resolution of the jet orifice was observed in the HMV and phase angle mapping measurements of the pulsating jet compared to the direct measurement of time averaged current for the same modulated jet system.

\section{Acknowledgements}

We thank RSC/EPSRC Analytical studentship for funding JK under grant EP/C011430/1 and the EPSRC (EP/D05849X/1) for funding for the high-speed camera.

Figure Legends 
Figure 1 Experimental setup. Note E represents working electrode, MS represents the minishaker, $\mathrm{J}$ represents the jet, A represents the accelerometer and $M$ represents the membrane. The dotted square shows the 'neck' region of the cell used for high-speed imaging of the fluid flow in the apparatus (see text).

Figure 2 (a) Images obtained from high-speed camera investigation of the motion of the fluid within the neck of the funnel. Note in this experiment the liquid phase consists of water with a red food dye (lower) and heptane (upper) at $20-23{ }^{\circ} \mathrm{C}$. The images were taken at $500 \mathrm{fps}$ while the shaker was driven at $16 \mathrm{~Hz}$. Every $4^{\text {th }}$ frame is shown with the appropriate time added to each individual frame with respect to the first frame in the sequence. The scale bar represents $2 \mathrm{~mm}$. (b) Plot showing the analysis of the actual displacement determined from the high-speed images ( $\circ)$ and the theoretical displacement from the accelerometer data $(\bullet)$ considering an oblate correction for the shape of the liquid/liquid interface.

Figure 3 Plot showing the current time history (-) of a $500 \mu \mathrm{m}$ diameter Pt electrode positioned at a distance of $1 \mathrm{~mm}(x=1 \mathrm{~mm})$ from the jet centre $(z=1$ $\mathrm{mm})$. The solution contained $5 \mathrm{mmol} \mathrm{dm}^{-3}\left[\mathrm{Fe}(\mathrm{CN})_{6}\right]^{3-}$ in $0.1 \mathrm{~mol} \mathrm{dm}^{-3} \mathrm{Sr}\left(\mathrm{NO}_{3}\right)_{2}$ electrolyte. The electrode was held at $-0.2 \mathrm{~V}$ vs. Ag. Included on the figure is the reference signal $\left(\mathrm{V}_{\text {ref }}-\right)$ and corresponding accelerometer signal $\left(\mathrm{V}_{\mathrm{acc}}-\right)$.

Figure 4 Schematic showing the progress of data acquisition and treatment. Here FG represents the function generator, AMP the amplifier supplying the mini shaker, $\mathrm{CF}$ the current follower and $\mathrm{ADC}$ the interface card. The grey boxes represent the software driven section of the approach while the black boxes the hardware employed. Note $V_{\text {adc }}$ represents the data collected by the 
ADC card as a function of time, $\left\langle V_{a d c}>\right.$ represents the time averaged ADC data, $\mathrm{V}_{\mathrm{c}}(\mathrm{t})$ represents the signal corrected for dc offset as a function of time, $\mathrm{V}_{\mathrm{cf}}(\mathrm{t})$ represents the result of multiplication of the dc corrected signal by the reference signal (corrected for phase shift) as a function of time, $\left\langle\mathrm{V}_{\mathrm{cf}}>\right.$ represents the time averaged signal, $\mathrm{G}_{\mathrm{cf}}$ represents the gain of the current follower and $\mathrm{E}_{0}$ represents the amplitude of the reference signal (here always $1 \mathrm{~V}$ ).

Figure 5 Plot showing the HMV signal $\left(\mathrm{i}_{\mathrm{HMV}},-\right)$ and average current signal ( $\mathrm{i}_{\mathrm{av}}$, -) as a function of electrode potential recorded at a $500 \mu \mathrm{m}$ diameter Pt electrode positioned at a distance of $1 \mathrm{~mm}(x=1 \mathrm{~mm})$ from the jet centre $(z=1$ $\mathrm{mm})$. The solution contained $5 \mathrm{mmol} \mathrm{dm}^{-3}\left[\mathrm{Fe}(\mathrm{CN})_{6}\right]^{3-}$ in $0.1 \mathrm{~mol} \mathrm{dm}^{-3} \mathrm{Sr}\left(\mathrm{NO}_{3}\right)_{2}$ electrolyte. The sweep was performed at $20 \mathrm{mV} \mathrm{s}^{-1}$. Included in the figure is the data with the jet running (labelled $\mathrm{J}$ ) and without the jet running (labelled $\mathrm{NJ})$.

Figure 6 (a) Plot showing the phase correction employed through the automated data collection software as a function of position. (b) Plot showing the mass transport-limited HMV signal ( $\mathrm{i}_{\text {нми }}$ ) at a $25 \mu \mathrm{m}$ diameter Pt electrode with an insulating glass sheath (diameter $=2 \mathrm{~mm}$ ) in a $0.1 \mathrm{~mol} \mathrm{dm}^{-3} \mathrm{Sr}\left(\mathrm{NO}_{3}\right)_{2}$ electrolyte containing $5 \mathrm{mmol} \mathrm{dm}^{-3}\left[\mathrm{Fe}(\mathrm{CN})_{6}\right]^{3-}$ recorded as a function of electrode position in the $x-y$ plane at a distance $z=1 \mathrm{~mm}$ from the funnel mouth. (c) Plot showing the time averaged dc current ( $\left.\mathrm{i}_{\mathrm{av}}\right)$ as a function of electrode position. The electrode was held at $-0.2 \mathrm{~V} v \mathrm{vs}$. Ag at all times. Note each plot is a composite of 960 points ( $200 \mu \mathrm{m}$ resolution) and is an average of 5 (2.5 s of data) measurements. 
Figure 7 (a) Plot showing the phase correction employed through the automated data collection software as a function of position. (b) Plot showing the mass transport-limited HMV signal (iнмv) at a $25 \mu \mathrm{m}$ diameter Pt electrode with an insulating glass sheath (diameter $=5.5 \mathrm{~mm}$ ) in a $0.1 \mathrm{~mol} \mathrm{dm}^{-3} \mathrm{Sr}\left(\mathrm{NO}_{3}\right)_{2}$ electrolyte containing $5 \mathrm{mmol} \mathrm{dm}^{-3}\left[\mathrm{Fe}(\mathrm{CN})_{6}\right]^{3-}$ recorded as a function of electrode position in the $x-y$ plane at a distance $z=1 \mathrm{~mm}$ from the funnel mouth. (c) Plot showing the time averaged dc current ( $\left.\mathrm{i}_{\mathrm{av}}\right)$ as a function of electrode position. The electrode was held at $-0.2 \mathrm{~V}$ vs. Ag at all times. The $z$ displacement was maintained at $1 \mathrm{~mm}$ from the tip of the funnel mouth. Note each plot is a composite of 960 points (200 $\mu \mathrm{m}$ resolution) and is an average of 5 (2.5 s of data) measurements.

Figure 8 Plots showing the transport-limited HMV signal ( $\mathrm{i}_{\text {HMV }}$ ) as a function of position of an electrode in the $x-z$ plane, where $x$ is horizontal and $z$ the vertical displacement. Here $y=0$ (centre of the funnel mouth). The data were recorded with $25 \mu \mathrm{m}$ diameter Pt electrodes (insulating glass sheath diameter of (a) $2 \mathrm{~mm}$ and (b) $5.5 \mathrm{~mm})$. The solution consisted of $0.1 \mathrm{~mol} \mathrm{dm}^{-3} \mathrm{Sr}\left(\mathrm{NO}_{3}\right)_{2}$ electrolyte containing $5 \mathrm{mmol} \mathrm{dm}^{-3}\left[\mathrm{Fe}(\mathrm{CN})_{6}\right]^{3-}$. The electrodes were held at $-0.2 \mathrm{~V}$ vs. Ag at all times. Note each plot is a composite of 960 points ( $200 \mu \mathrm{m}$ resolution) and is an average of 5 ( $2.5 \mathrm{~s}$ of data) measurements.

Figure 9 (a) Plot showing the phase correction employed through the automated data collection software as a function of position. (b) Plot showing the mass transport-limited HMV signal (iнмv) at a $500 \mu \mathrm{m}$ diameter Pt electrode with an insulating glass sheath (diameter $=5.0 \mathrm{~mm}$ ) in a $0.1 \mathrm{~mol} \mathrm{dm}^{-3} \mathrm{Sr}\left(\mathrm{NO}_{3}\right)_{2}$ 
electrolyte containing $5 \mathrm{mmol} \mathrm{dm}^{-3}\left[\mathrm{Fe}(\mathrm{CN})_{6}\right]^{3-}$ recorded as a function of electrode position in the $x-y$ plane at a distance $z=1 \mathrm{~mm}$ from the funnel mouth. (c) Plot showing the time averaged dc current ( $\left.\mathrm{i}_{\mathrm{av}}\right)$ as a function of electrode position. The electrode was held at $-0.2 \mathrm{~V}$ vs. Ag at all times. The $\mathrm{z}$ displacement was maintained at $1 \mathrm{~mm}$ from the tip of the funnel mouth. Note each plot is a composite of 960 points ( $200 \mu \mathrm{m}$ resolution) and are an average of 5 ( $2.5 \mathrm{~s}$ of data) measurements. 


\section{References}

1. C. M. A. Brett and A. M. O. Brett, Electroanalysis, Oxford science publications, Oxford, 1998.

2. Z. F. Ding, B. M. Quinn, S. K. Haram, L. E. Pell, B. A. Korgel and A. J. Bard, Science, 296 (2002) 1293-1297.

3. I. Turyan, M. Atiya and D. Mandler, Electroanalysis, 13 (2001) 653-659.

4. J. V. Macpherson, Electroanalysis, 12 (2000) 1001-1011.

5. B. Miller and S. Bruckenstein, Annals of the New York Academy of Sciences, 404 (1983) 473-474.

6. K. Tokuda, S. Bruckenstein and B. Miller, Journal of the Electrochemical Society, 122 (1975) 1316-1322.

7. K. Tokuda, S. Bruckenstein and B. Miller, Journal of the Electrochemical Society, 122 (1975) C110-C110.

8. B. Miller and S. Bruckenstein, Analytical Chemistry, 46 (1974) 2026-2033.

9. B. Miller and S. Bruckenstein, Journal of the Electrochemical Society, 121 (1974) 1558-1562.

10. B. Miller and S. Bruckenstein, Abstracts of Papers of the American Chemical Society, (1974) 141-141.

11. B. Miller and S. Bruckenstein, Journal of the Electrochemical Society, 121 (1974) C124-C124.

12. W. J. Blaedel and J. Wang, Analytical Chemistry, 52 (1980) 1697-1700.

13. W. J. Blaedel and J. Wang, Analytica Chimica Acta, 116 (1980) 315-322.

14. W. J. Blaedel and Z. Yim, Analytical Chemistry, 52 (1980) 564-566.

15. W. J. Blaedel and R. C. Engstrom, Analytical Chemistry, 50 (1978) 476-479.

16. S. A. Schuette and R. L. McCreery, Analytical Chemistry, 58 (1986) 1778-1782.

17. D. E. Williams, K. Ellis, A. Colville, S. J. Dennison, G. Laguillo and J. Larsen, Journal of Electroanalytical Chemistry, 432 (1997) 159-169.

18. H. D. Dewald and B. A. Peterson, Analytical Chemistry, 62 (1990) 779-782.

19. E. L. Cooper and L. A. Coury, Journal of the Electrochemical Society, 145 (1998) 1994-1999.

20. N. Simjee, P. R. Unwin and J. V. Macpherson, Electroanalysis, 15 (2003) 14451452.

21. J. V. Macpherson and P. R. Unwin, Analytical Chemistry, 71 (1999) 4642-4648. 
22. J. V. Macpherson and P. R. Unwin, Analytical Chemistry, 71 (1999) 2939-2944.

23. W. Merzkirch, Flow Visualization, 2nd Edition, Academic Press, Orlando, 1987.

24. T. Seno, S. Kageyama and R. Ito, Journal of Chemical Engineering of Japan, 21 (1988) $1-5$.

25. T. Seno, S. Kageyama and R. Ito, Journal of Chemical Engineering of Japan, 20 (1987) 128-133.

26. E. W. Weisstein, "Volume." From MathWorld--A Wolfram Web Resource. http://mathworld.wolfram.com/Volume.html

27. J. Hellemans, P. Forrez and R. Dewilde, American Journal of Physics, 48 (1980) 254-256.

28. J. H. Scofield, American Journal of Physics, 62 (1994) 129-133.

29. J. L. Melville, B. A. Coles, R. G. Compton, N. Simjee, J. V. Macpherson and P. R. Unwin, Journal of Physical Chemistry B, 107 (2003) 379-386.

30. J. V. Macpherson, M. A. Beeston and P. R. Unwin, Journal of the Chemical Society-Faraday Transactions, 91 (1995) 899-904. 


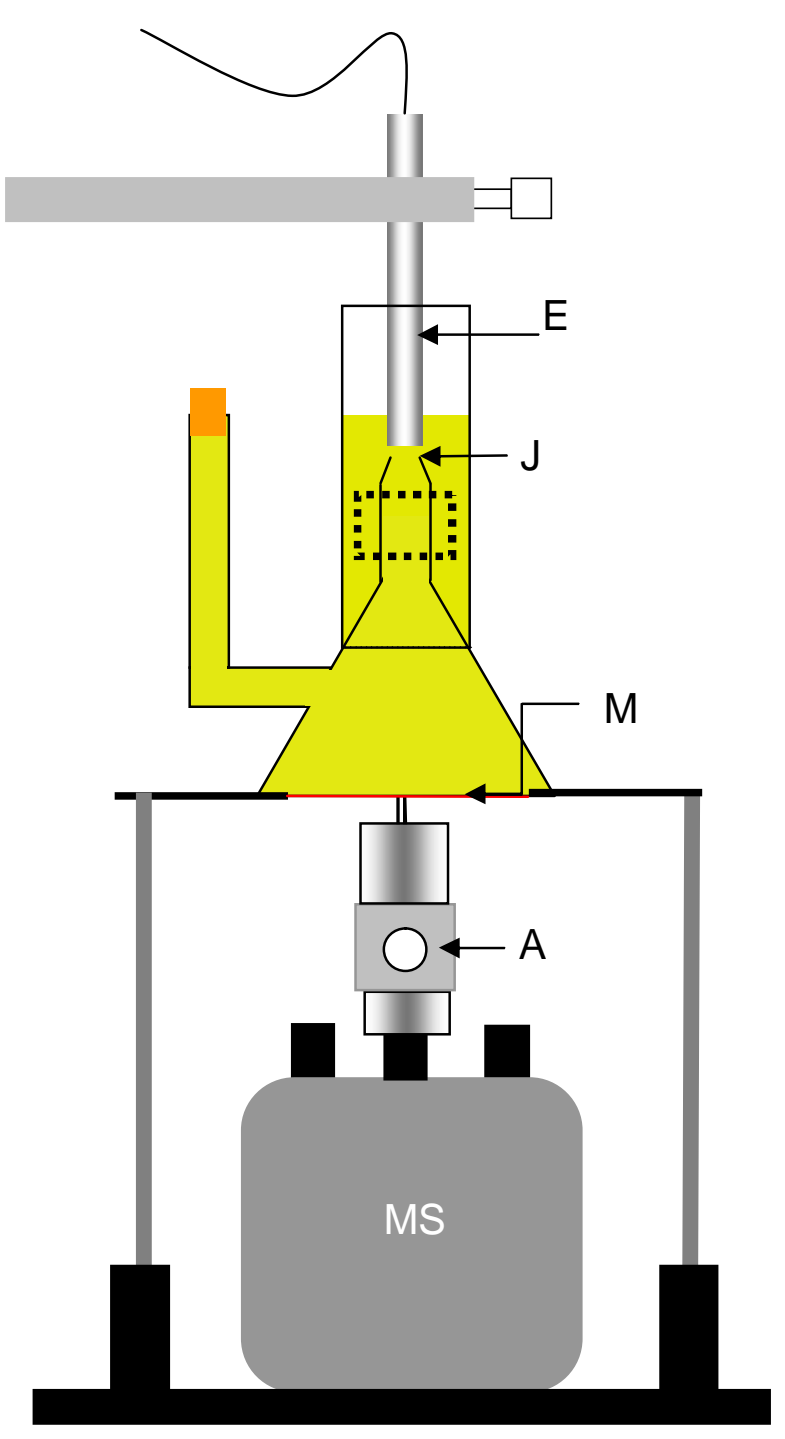

Figure 1. 
(a)

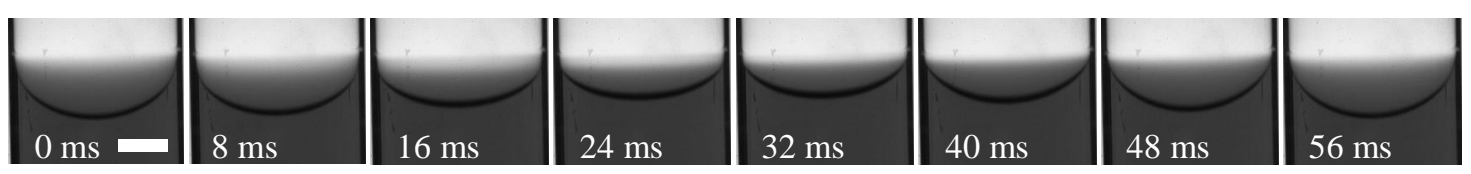

(b)

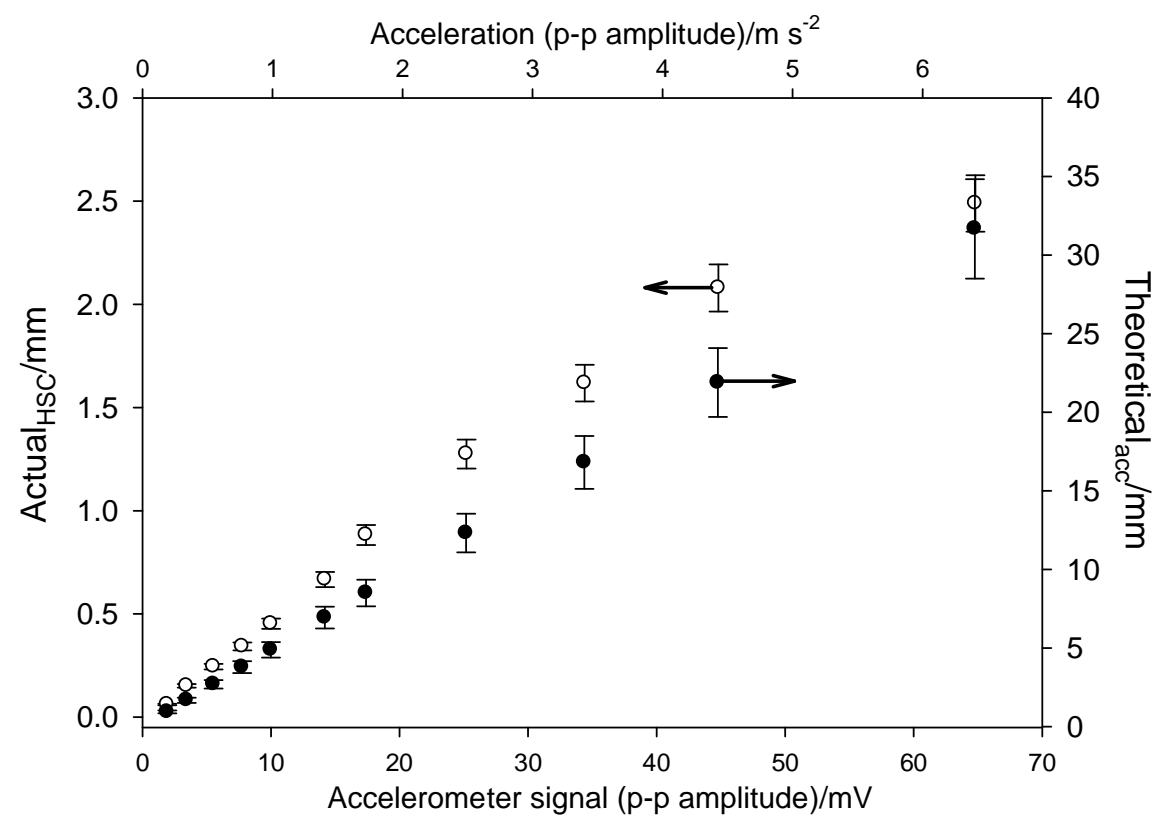

Figure 2 


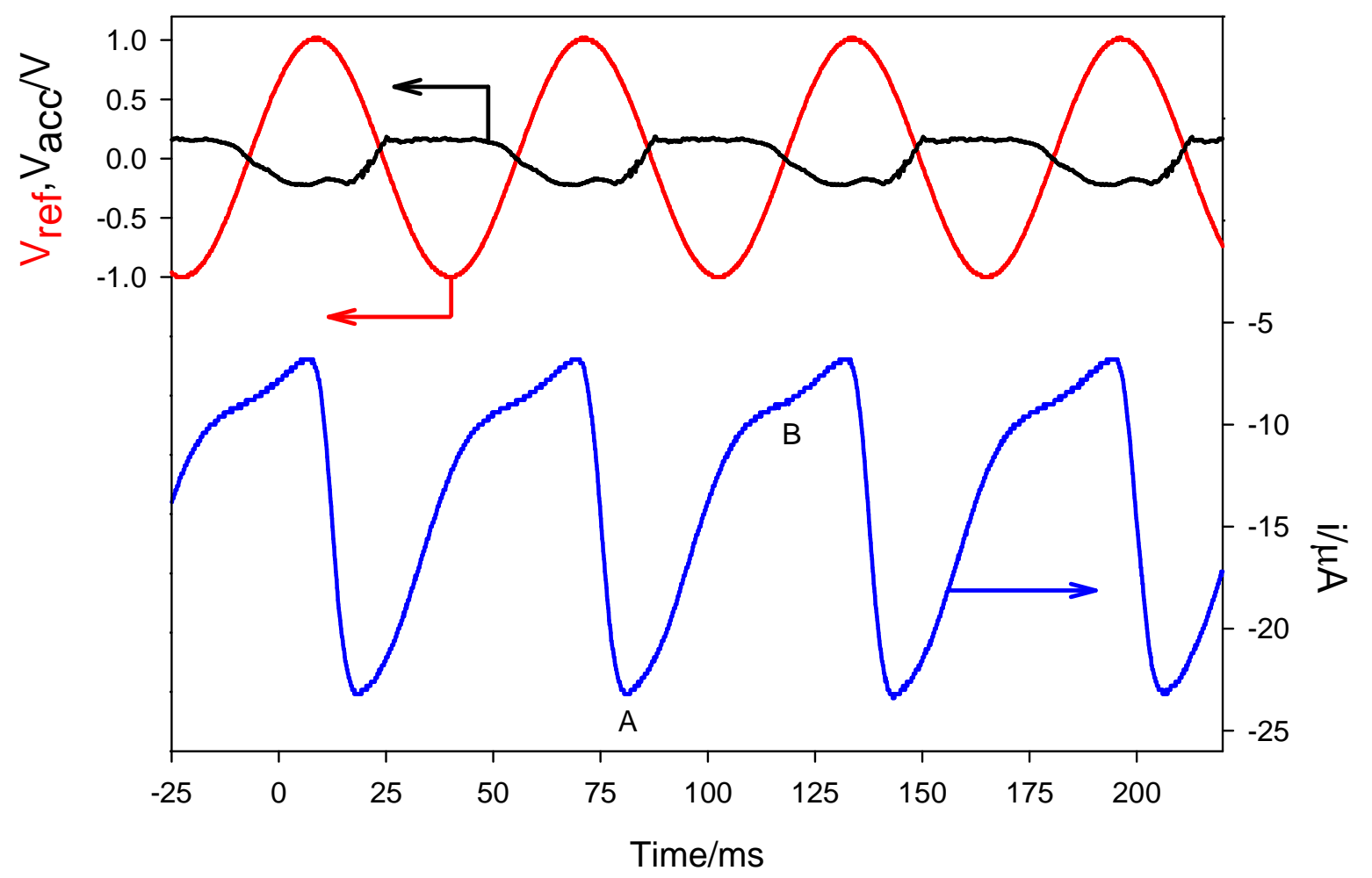

Figure 3. 


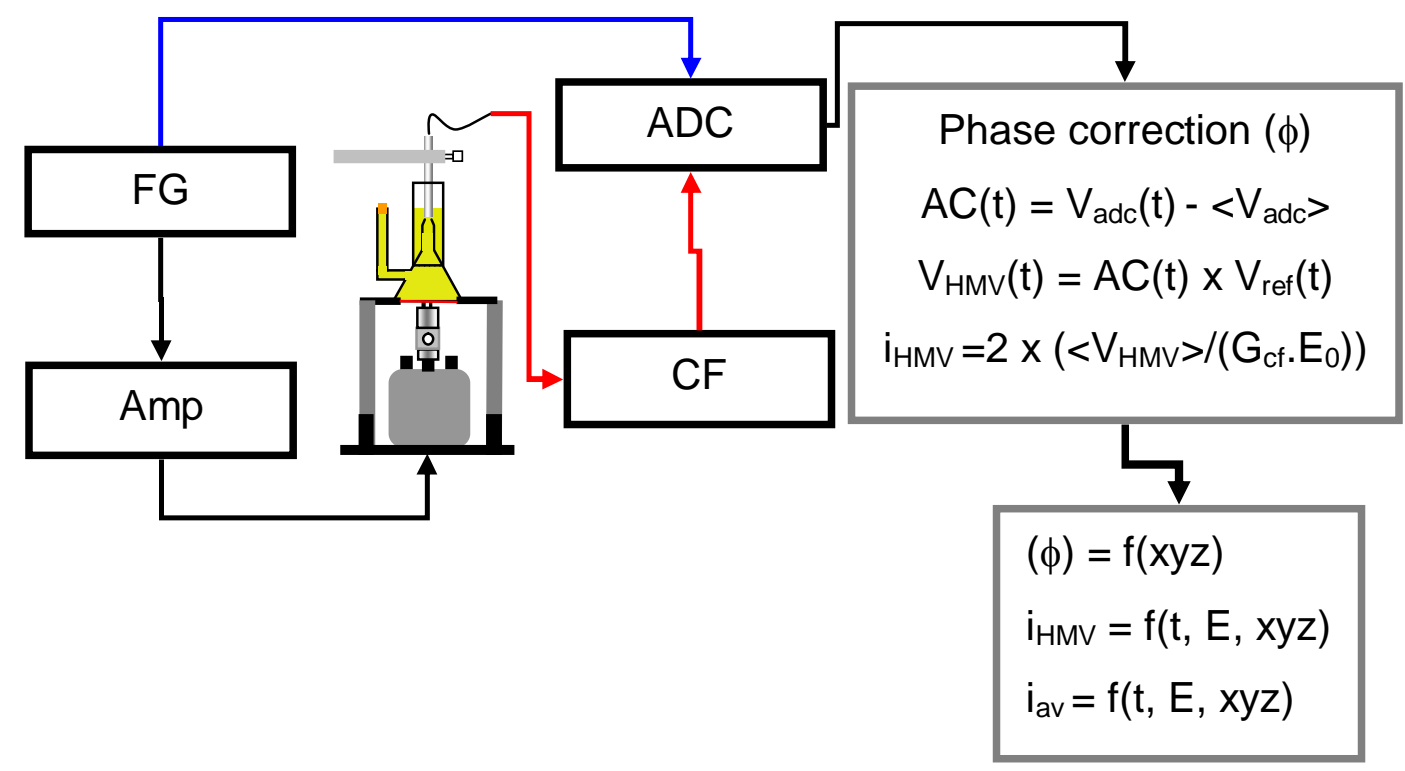

Figure 4. 


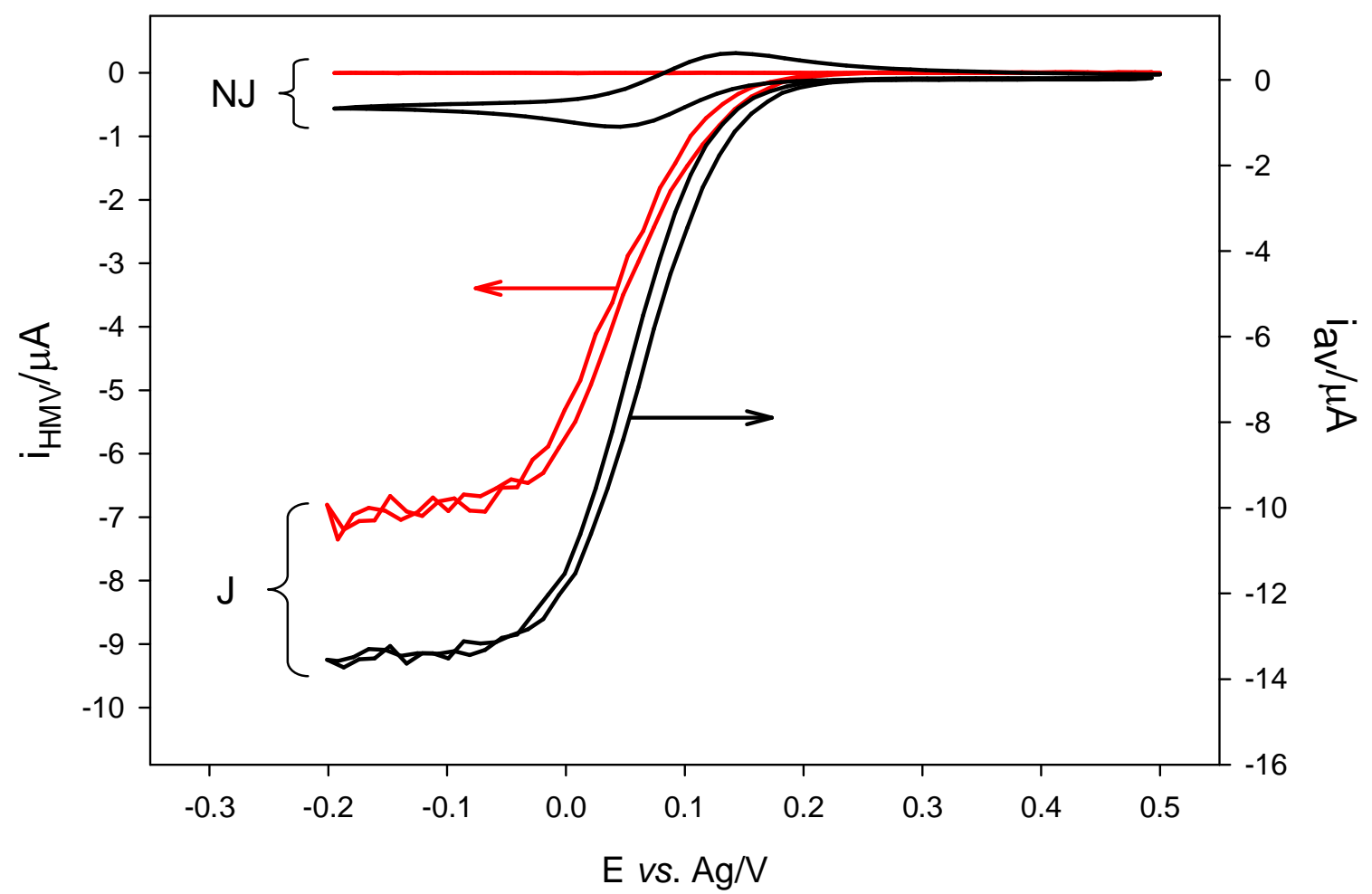

Figure 5. 

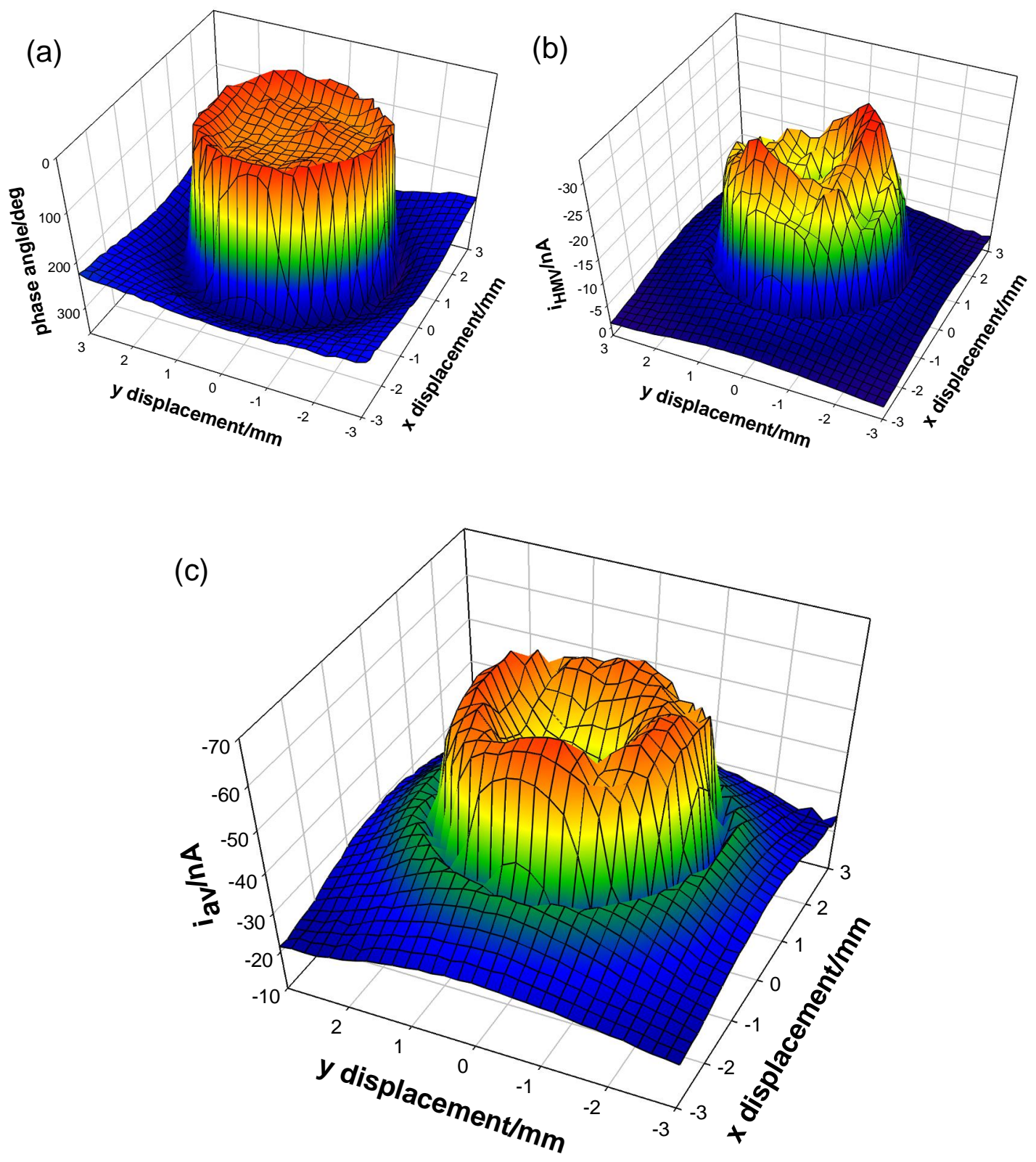

Figure 6 

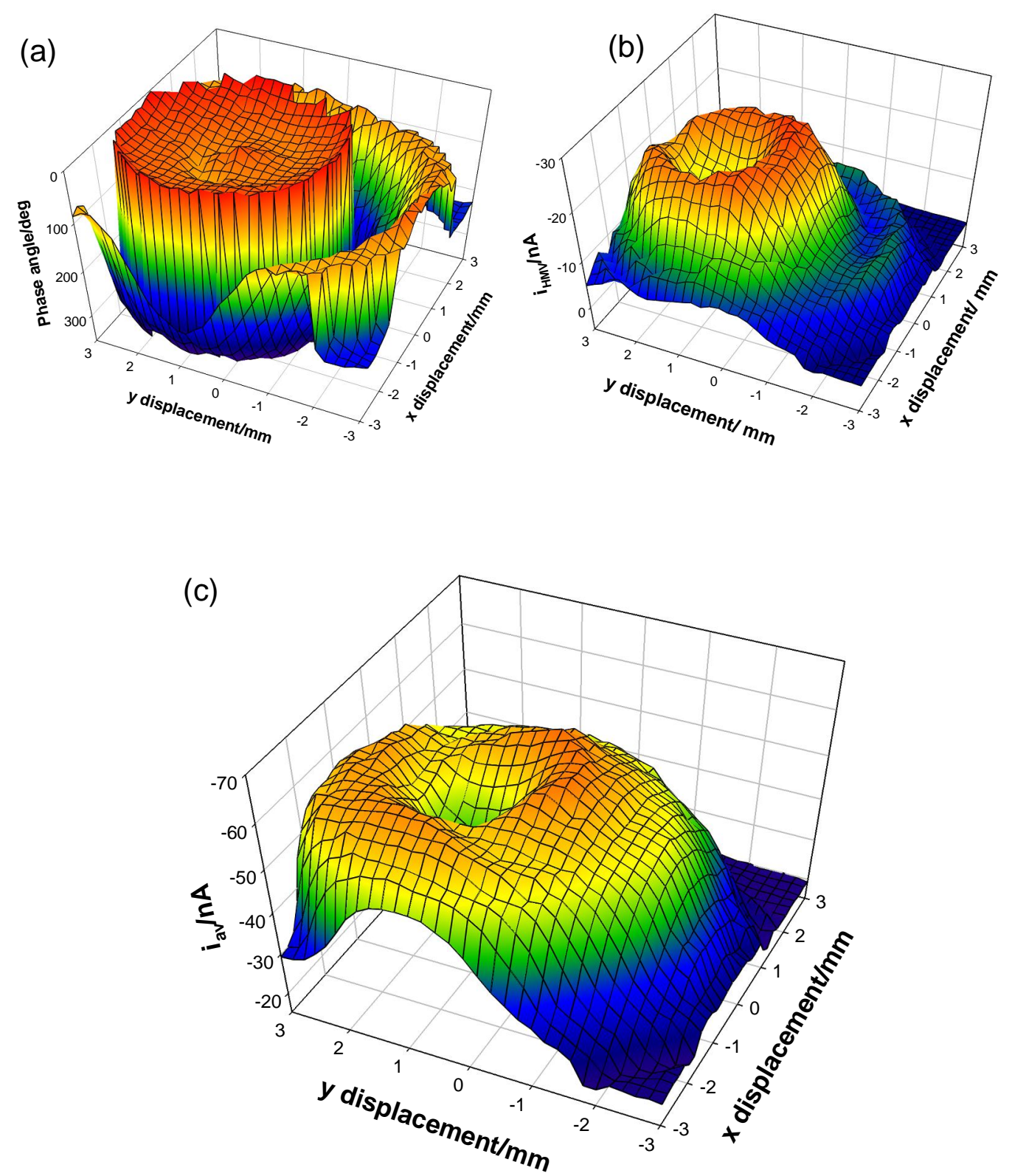

Figure 7 
(a)

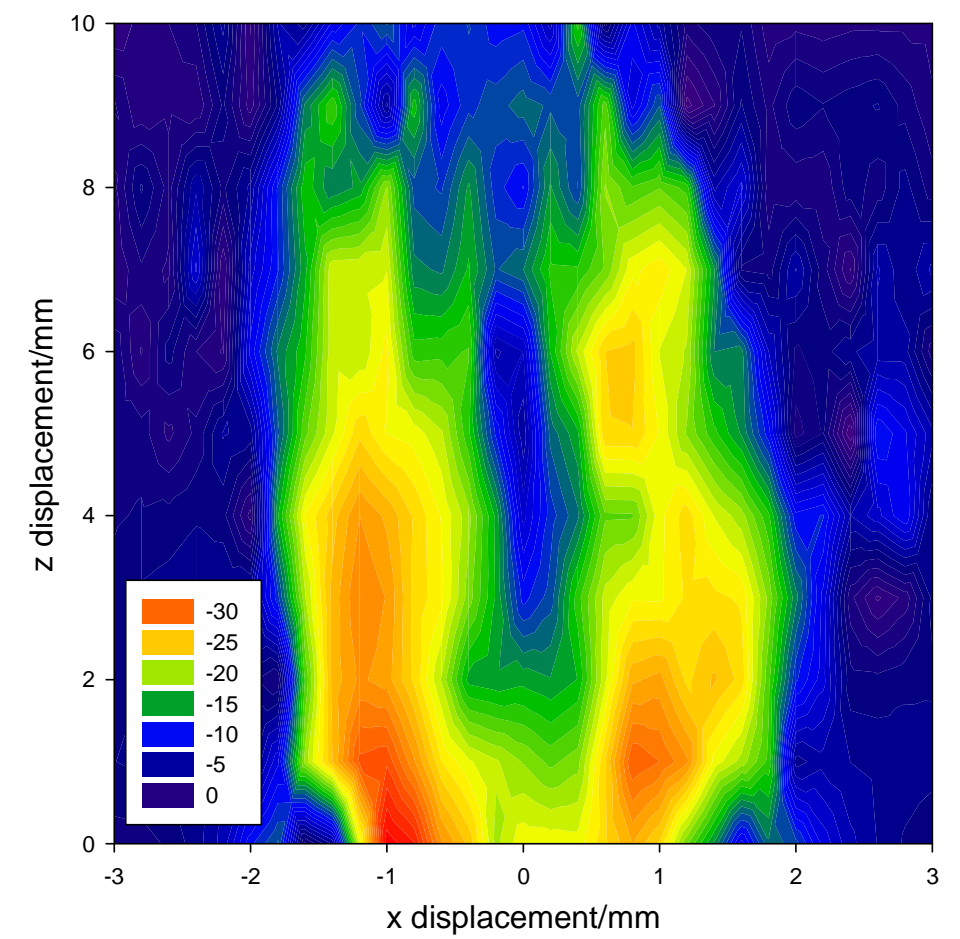

(b)

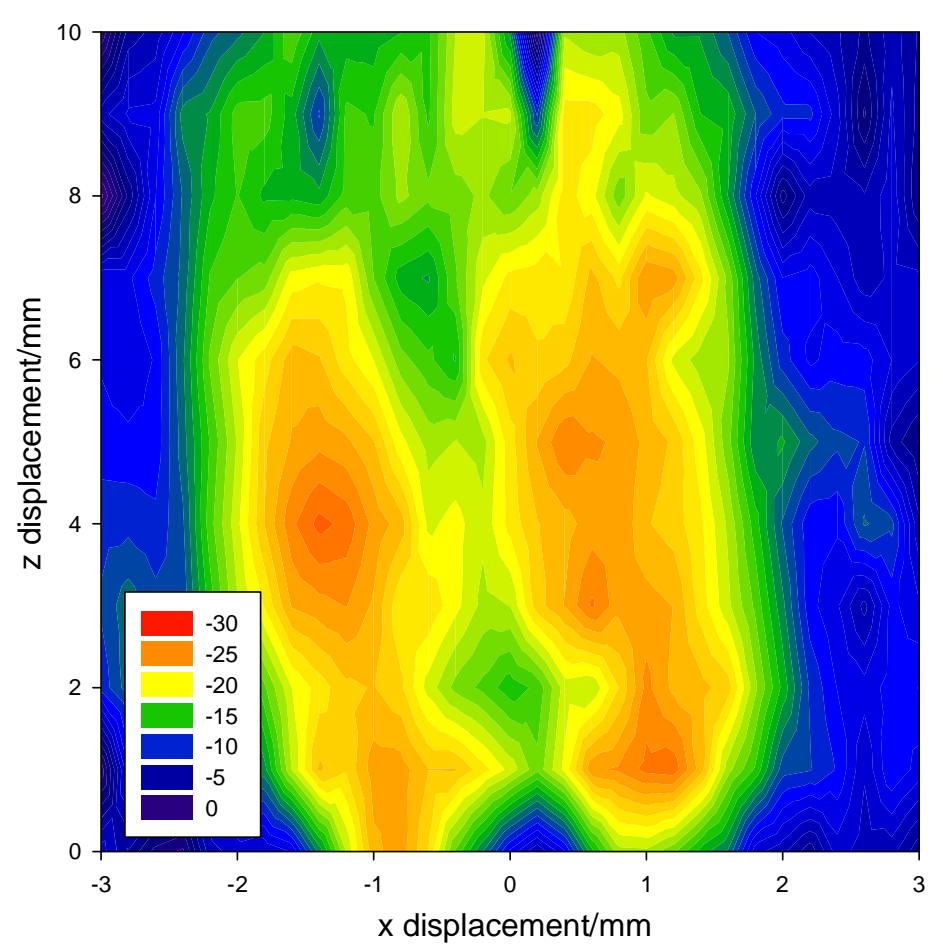

Figure 8 
(a)
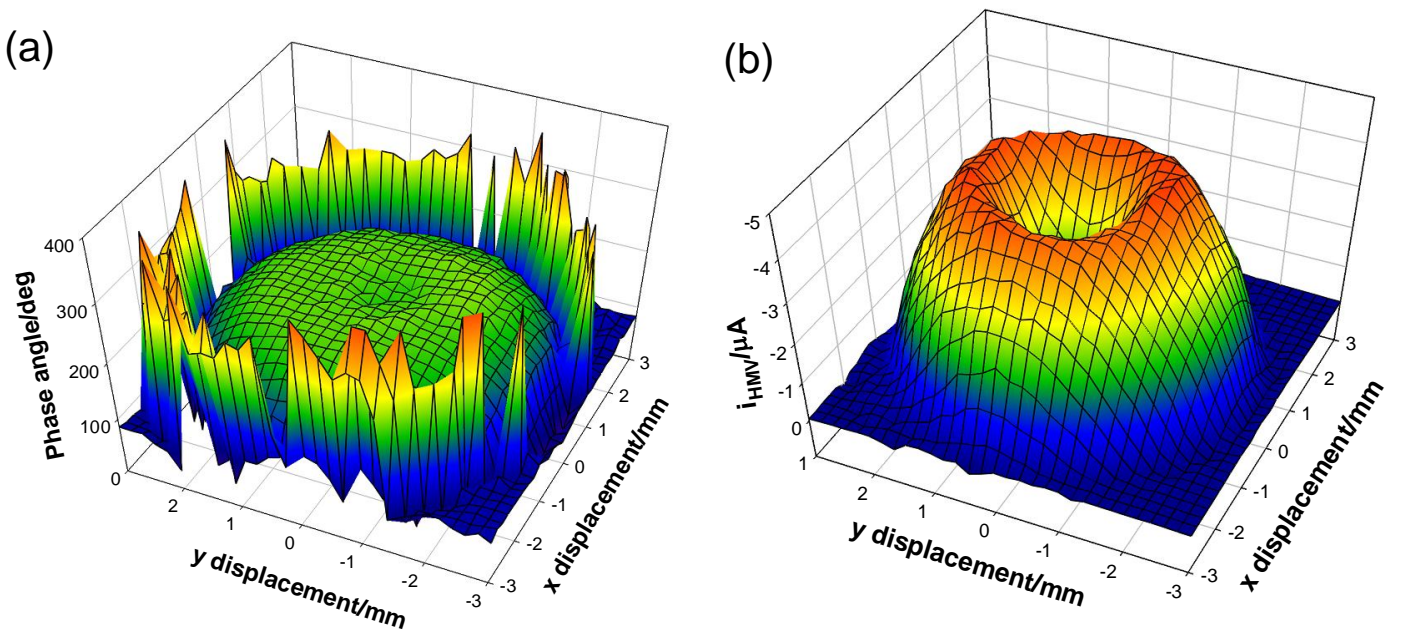

(c)

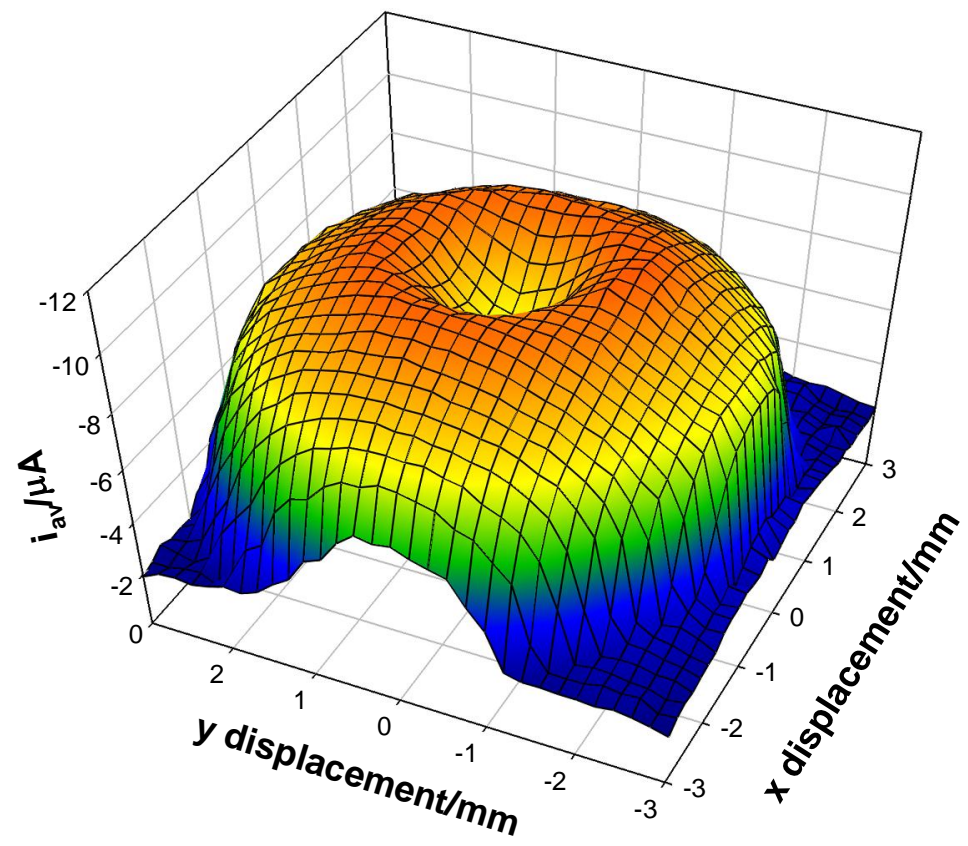

Figure 9 